\title{
Single anastomosis gastric bypass: A novel bariatric procedure
}

\author{
M. Mahir Özmen, ${ }^{1}$ Tevfık Tolga Şahin, ${ }^{1}$ C. Emir Güldoğan ${ }^{2}$ \\ 'Department of General Surgery, Hacettepe University Faculty of Medicine, Ankara, Turkey \\ 2Department of General Surgery, Ankara Numune Training and Research Hospital, Ankara, Turkey
}

\begin{abstract}
Since Rutledge developed single anastomosis gastric bypass in 1990, it has been popularized by surgeons in Europe as procedure of choice in the morbidly obese. It involves creating gastric pouch of $12 \mathrm{~cm}$ and antecolic loop gastroenterostomy $200 \mathrm{~cm}$ distal to the ligament of Treitz. It has comparable excess body weight loss rate to Roux-en-Y gastric bypass, and safety profile is better. This review summarized technical details and key points in performing safe procedure and outlined results of key, high volume series in terms of outcome and technical feasibility.
\end{abstract}

Keywords: Bariatric and metabolic surgery; morbid obesity; single anastomosis gastric bypass.

\section{Introduction}

Foundations of gastric bypass as a bariatric procedure was first developed in 1966 by Mason and Ito. ${ }^{[1]}$ Initially, a transverse gastric pouch was created and loop gastroenterostomy line was high in the upper abdomen. ${ }^{[1]}$ Anastomosis formation was technically difficult, and since it was close to the esophagus, these procedures were complicated with alkaline reflux esophagitis. ${ }^{[2]}$ In time, dilatation of the fundus occurred, leading to the failure of the technique as a bariatric procedure. ${ }^{[3]}$

In 1990, Rutledge et al. first described single anastomosis gastric bypass (Minigastric bypass; LSAG) as an alternative novel bariatric procedure. ${ }^{[4]}$ In this procedure, gastric pouch was created parallel to the lesser curvature of the stomach. Loop gastroenterostomy was created at the lower part of the gastric pouch. ${ }^{[5]}$ Furthermore, the fundus was also excluded from the gastric pouch. ${ }^{[6]}$ All of these technical properties made the technique simple and reversible when compared to other malabsorptive procedures. ${ }^{[7]}$

It was first applied laparoscopically in 2002 and has been popularized since 2006 as an effective bariatric procedure. ${ }^{[8]}$ Ever since, there has been large volume data with patient numbers reaching tens of thousands. The results of the technique were comparable to Roux-en-Y Gastric bypass which is still considered the golden standard technique. ${ }^{[9]}$

The aim of the present study is to review the current literature in terms of the technical aspects of the procedure, and at the later part of the manuscript, results of some major studies as well as the experience of our center will be demonstrated. 
Table 1. Steps of the laparoscopic single anastomosis gastric bypass

\section{Patient position \\ Pneumoperitoneum \\ Trocar placements \\ Liver retraction \\ Forming the gastric pouch \\ Creating the gastroenterostomy}

\section{Procedure}

The procedure will be explained in subsections shown in Table 1. Controversies, pitfalls and technical variations will be discussed.

\section{Patient Position}

The patient is placed in supine position with the legs spread apart (French position) (Figure 1). The operating table could be tilted in $30^{\circ}$ reverse Trendelenburg position as the surgeon needs. The operating room should be wide enough for manipulating the table, and the operating table should be able to with stand extreme weights of the patient. Technical variations regarding patient positioning consists positioning the patient in supine position without spreading the legs. In this scenario, the operating surgeon stands on the right side of the patient. ${ }^{[8]}$

\section{Pneumoperitoneum}

Insufflation is usually performed from the first trocar insertion site. It can be over or at the umbilical region of the patient. However, there are two pitfalls during the establishment of the pneumoperitoneum. The first important point is that obese individuals have a wider upper abdomen, and if the initial trocar is placed low near the umbilicus, the dissection of the upper abdomen can be more difficult. Second pitfall is bypassing the falciform ligament. In obese individuals, the falciform ligament can be hypertrophied due to adipose tissue hyperplasia. If insufflation is to be carried out near the midline, the falciform ligament can make insufflation and initial trocar insertion technically challenging. We prefer establishing the pneumoperitoneum by Veress needle inserted $10 \mathrm{~cm}$ below the xiphoid process near the midline but $1 \mathrm{~cm}$ deviated to the left in order to avoid insertion into the falciform ligament (Figure 2). Alternatively, the first trocar can be inserted under direct vision by Hasson's technique; however, the insertion site should be carefully selected.

\section{Trocar Placement}

Four to six trocars can be used in order to perform the operation. We usually prefer four trocars routinely. In case we encounter a difficulty in dissection, only then we insert additional $5 \mathrm{~mm}$ trocars for traction and assistance. The localization of our trocars is (Figure 3a):

1. For establishing insufflation: Veress/Hasson's trocar is inserted through the left deviated midline trocar which is $10 \mathrm{~cm}$ away from the xyphoid process. Here, 10 or $12 \mathrm{~mm}$ ports are used and we usually prefer $30^{\circ}$ scope to aid the operation.

2. Subxyphoid trocar is inserted under direct vision and liver retraction is inserted ( 5 or $10 \mathrm{~mm}$ trocars; determined according to the liver retraction method).

3. $12 \mathrm{~mm}$ ports are inserted from the right and left midclavicular region near the camera port which are used to
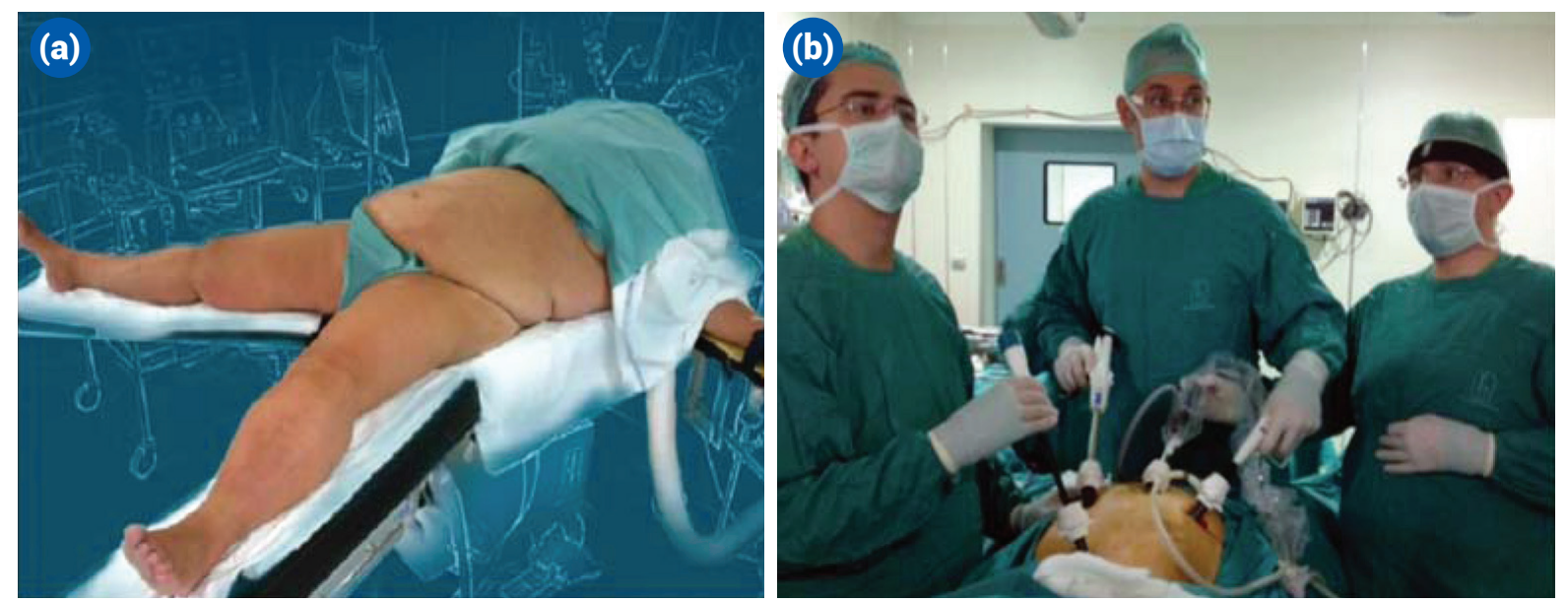

Figure 1. Patient positioning. (a) Patient lying down in supine position; (b) The surgeon is between the legs in 'French' position. 

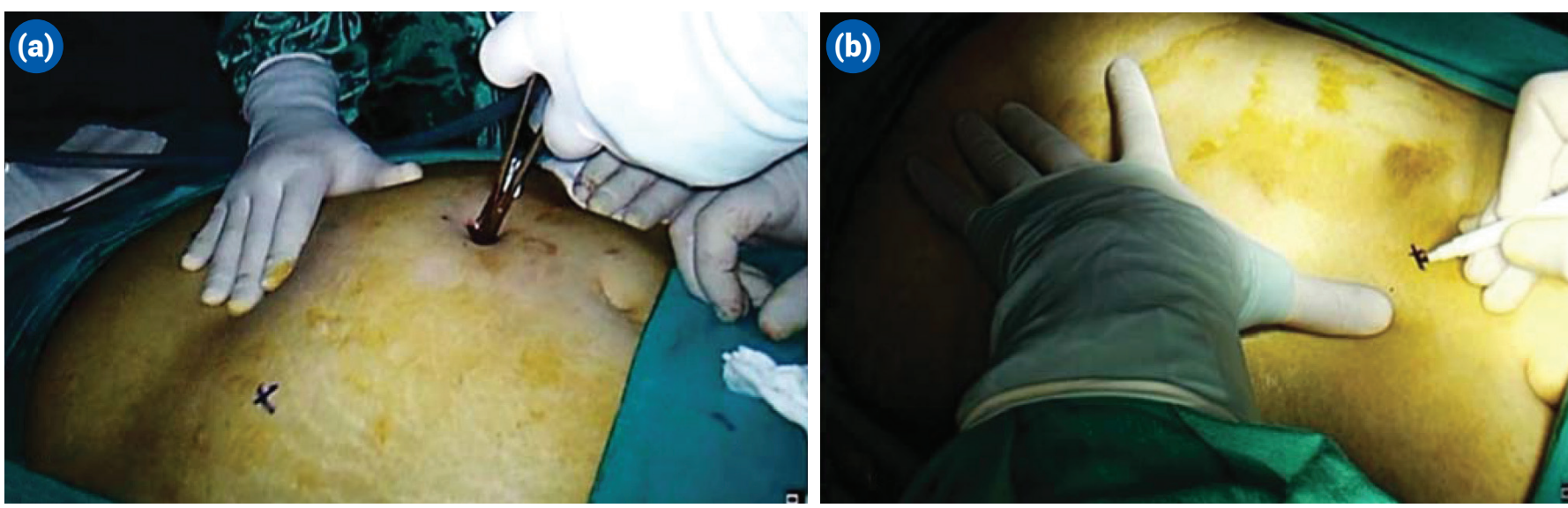

Figure 2. Establishing the pneumoperitoneum. (a) Establishing the pneumoperitoneum; (b) Veress needle is inserted $10 \mathrm{~cm}$ below the xiphoid process deviated $1 \mathrm{~cm}$ to the left of the midline (to avoid the falciform ligament).
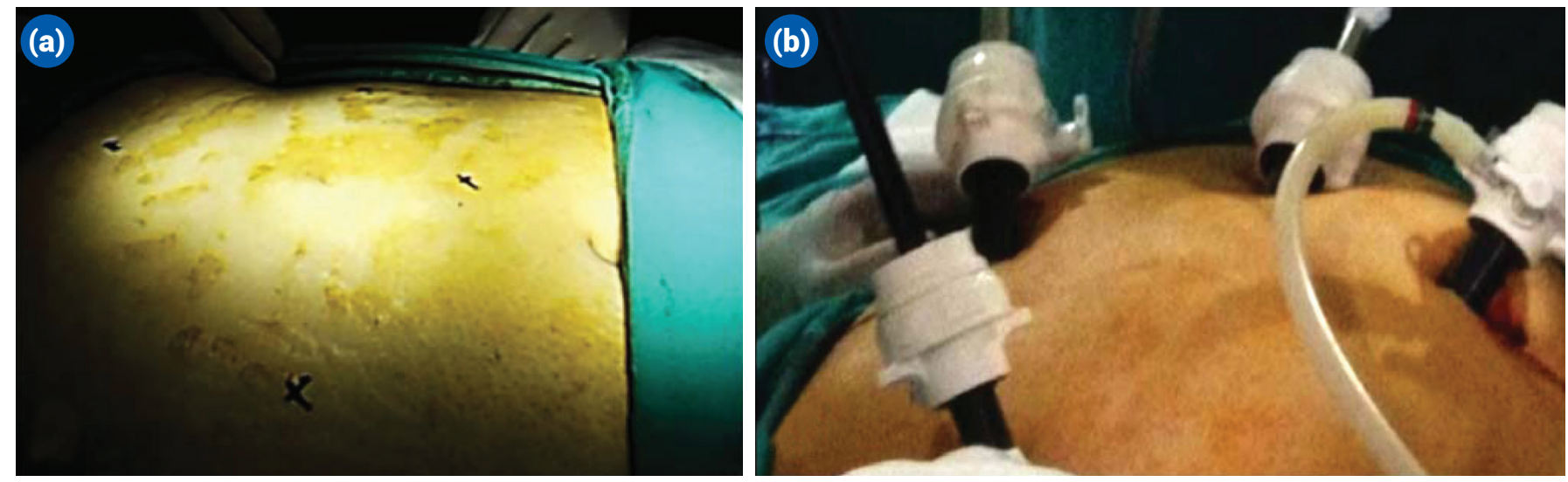

Figure 3. Placement of the trocars. (a) The preferred places of the trocars. (b) The positions of the trocars after insertion.

insert the staplers and the intestinal clamps.

In Figure 3b, the appearance after insertion of all the trocars is visualized. The authors prefer performing the operation with four trocars. However, if any difficulty in dissection is encountered, then, right and left lateral hypochondriac region can be used to insert $5 \mathrm{~mm}$ port for traction and exposure purposes.

\section{Liver Retraction}

Liver is a major obstacle during upper abdominal operations. Unless the patient consumes a low calorie liquid diet prior to bariatric procedure, the surgeon is usually faced with enlarged liver obscuring the exposure. Usually, liver retraction is performed through access created from the subxyphoid area. Fan shaped hand held retractor, Nathanson's liver retractor,and peanut retractor can be used according to surgeons' preference. In cases of enlarged liver observed upon access to the abdomen, localization of the trocars can be switched to lower locations than originally used. The authors' prefer using the Nathanson's retractor since it is inserted from the $5 \mathrm{~mm}$ port which reduces wound size, pain and risk of hernia. Moreover, it reduces personnel need. Figure 4 shows a case where $10 \mathrm{~mm}$ fan shaped liver retractor has been used. In suitable cases with small livers, a suture passing through the round ligament of the liver may also suffice to provide suitable retraction for adequate exposure.

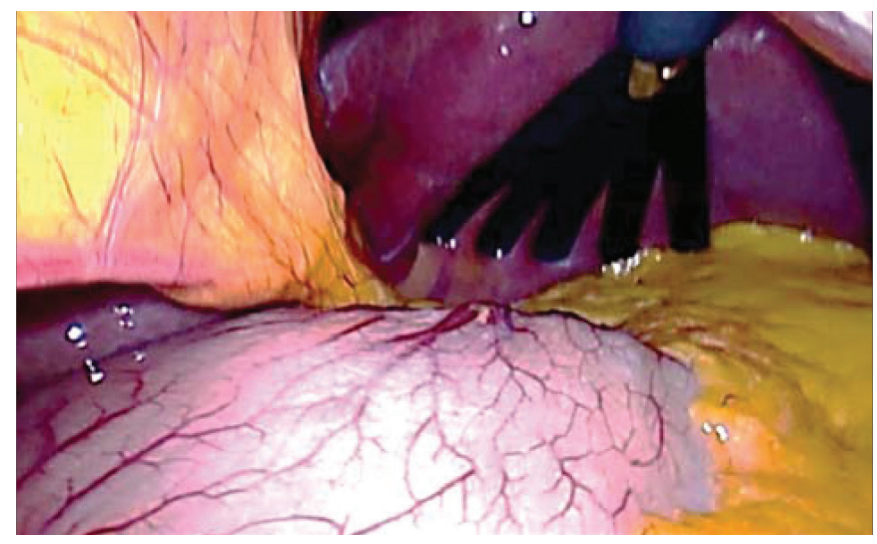

Figure 4. A case in which 'fan shaped' liver retractor is used. 


\section{Forming Gastric Pouch}

The main goal here is to make $12 \mathrm{~cm}$ long narrow gastric tube starting from incisuraangularis of the lesser curvature. The dissection usually extends up to the angle of His. There are also three main pitfalls for this technique. Initially, while forming the tube, the surgeon must enter the lesser omental sac through a perigastric dissection at the incisuraangularis. During this maneuver, there can be damage to the adjacent organs like the pancreas or can cause extensive bleeding, and the surgeon must be ready for such surprises. A meticulous dissection is imperative, and it is obligatory to visualize every anatomic detail of the dissection until the lesser omental sac is accessed safely (Figure 5a).
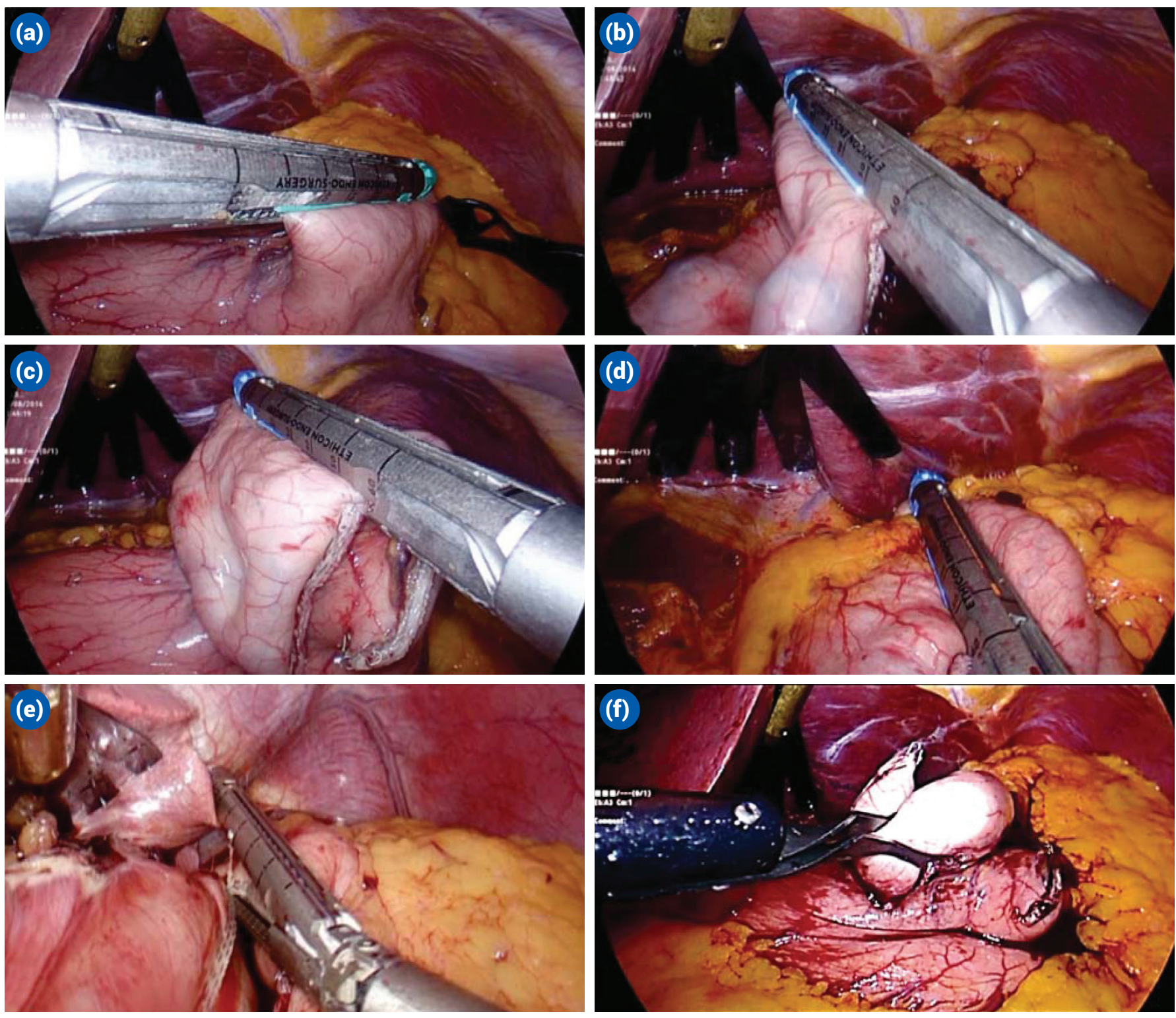

Figure 5. (a) Transection of the stomach (b-e). Formation of the tube towards the angle of His (f). The formed gastric pouch.
Another problem is the formation completion of the gastric tube near the angle of His. The authors prefer starting the dissection from the angle of His and mobilize the stomach by dissecting the gastrosplenic ligament and visualizing the left crura of the diaphragm. This step is crucial for it greatly simplifies the application of the stapler towards the end of completion of the gastric tube. Crucial point in the angle of his is first dissecting the ligament carefully and preserving the short gastric vessel in order to preserve the vascular supply of the stomach. Therefore, we suggest that unless there is extensive bleeding from the site, no clips or ligature shall be applied during the dissection of this area. Another crucial point is visualizing the left crura. After the dissection of the angle of His, it is 
much easier to apply the stapling device, and especially, it makes the completion of the gastric tube much easier (Figures $5 \mathrm{~b}-\mathrm{f}$ ). We usually prefer medium thickness sta- plers in order to form the gastric tube, which are purple for Covidien Triple Staple Technology (Mineapolis, USA), gold for the Ethicon endostaplers (New Jersey, USA) or Co-
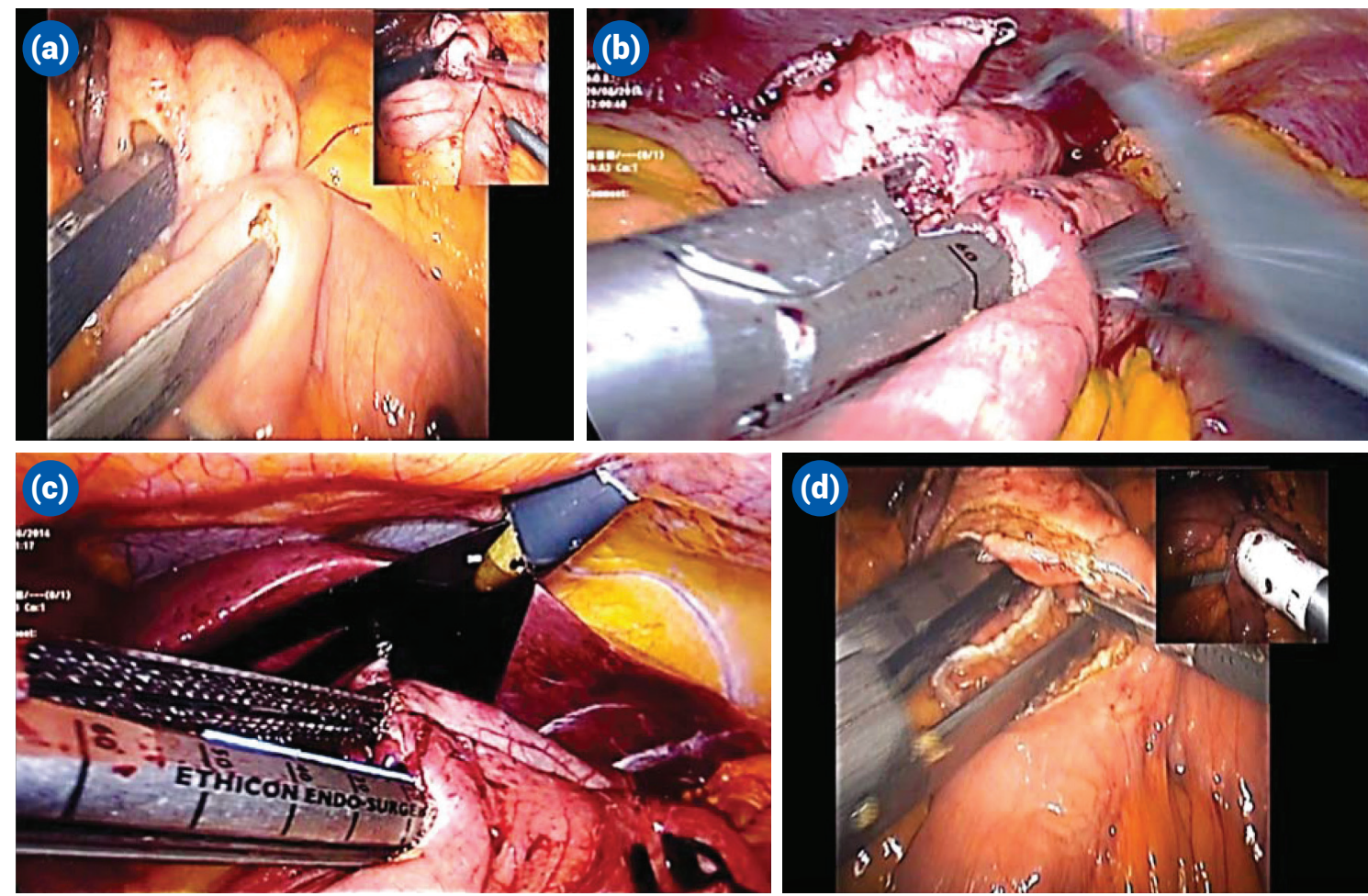

Figure 6. (a) Creation of the gastroenterostomy line by stapler (The in-lay shows the insertion of the OrVil). (b, c) The completed gastroenterostomy line by the endostapler. (d) Shows different methods of gastroenterostomy (in-lay shows deployment of circular stapler).
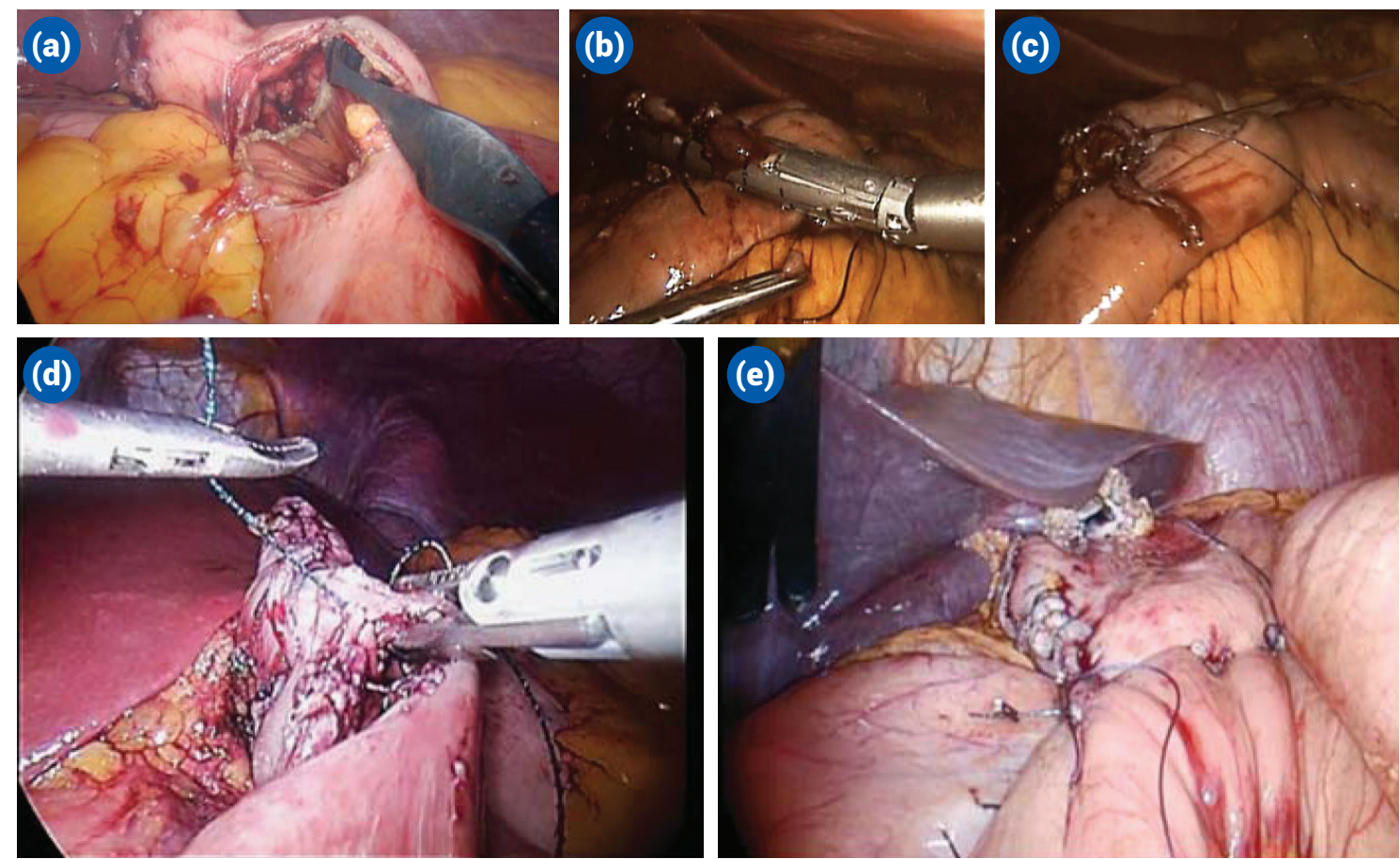

Figure 7. Closure techniques of the gastric stoma after anastomosis. (a) Gastric hole following formation of the anastomosis. (b, c) Closure of the gastric hole with stapler. (d, e) Closure with pre-tied suture. 


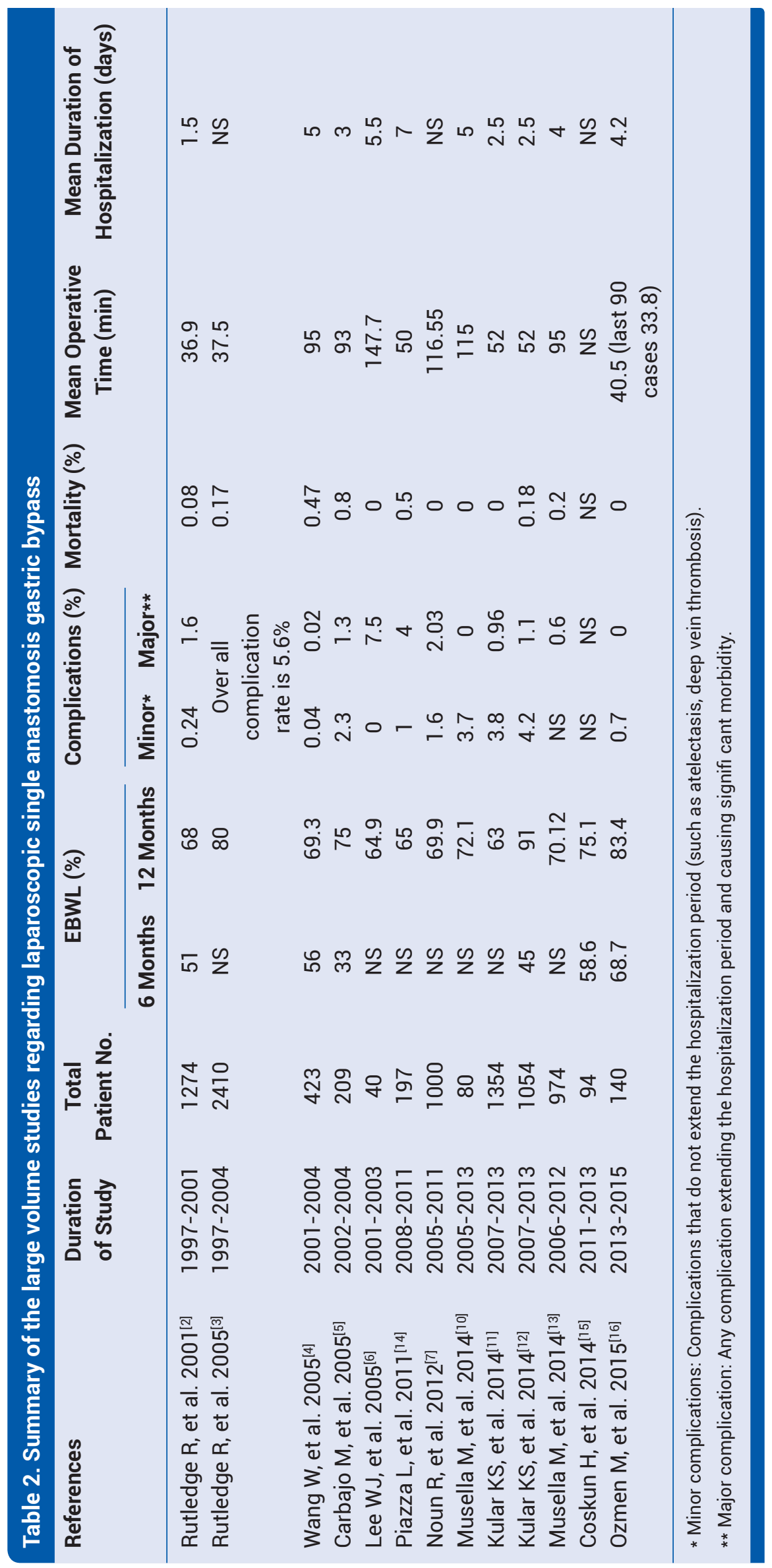


vidien Or-Vil circular stapling system (Mineapolis, USA).

Another pitfall in the formation of the gastric tube is the lateral traction of the corpus of the stomach in order to prevent laving gastric tissue on the posterior aspect. ${ }^{[8]}$ Not only does this maneuver increase the success of the procedure but it also decreases the incidence of twist in the gastric pouch reducing the functional obstruction. ${ }^{[8,9]}$

\section{Creation of Gastroenterostomy}

Creation of gastroenterostomy is usually made two hundred centimeters distal to the ligament of Trietz. In case of co-morbidities such as diabetes or hypertension, anastomosis is usually made to the two hundred fiftieth centimeter. We prefer making gastroenterostomy anastomosis with medium thick staplers as well. This phase of the operation is fairly straight forward; however, it needs surgeon expertise to search and find the ligament of Trietz. It is the authors' preference to put the patient to a neutral position with a $30^{\circ}$ tilt towards the patient's right side, which keeps the free intestinal loops, omentum, and transverse colon away from the ligament of Trietz and makes the recognition relatively easier (Figure 6).

Following the formation of the gastroenterostomy line, the opening can be closed by another cartridge with medium height or running pre-teid sutures (Figure 7).

\section{Discussion}

Single anastomosis gastric bypass, pioneered by Rutledge as mini-gastric bypass, is an operative procedure including a long narrow gastric tube in conjunction with an antecolic loop gastroenterostomy anastomosis. ${ }^{[2]}$ It is more advantageous than Roux-en-Y gastric bypass because it enables a tension free gastroenterostomy anastomosis, and the technique avoids formation of a Roux-en-Y limb. All of these characteristics providea lower complication rate when compared to other alternatives. ${ }^{[2,4,7]}$ A study by Lee et al. has shown excellent long-term results in terms of weight loss and low complication profile. ${ }^{[6]}$ It can be regarded an alternative to Roux-en-Y gastric bypass due to better reproducibility, good outcome, lower mortality and morbidity. ${ }^{[7]}$ The following subsections will be evaluating excess body weight loss rates, complication rates, and duration of hospitalization. The metabolic effects are summarized in another chapter in the present issue of the article, but it is a popular research subject studied by various researchers. ${ }^{[7-16]}$ All data are summarized in Table 2.

\section{Excess Body Weight Loss Rates}

Excess body weight loss rates have been reported to be $60 \%$ to $75 \%$ in different reports. The success of the LSAGB is attributable to the malabsorbtive properties of the procedure. Few studies have evaluated the efficacy of LSAGB over Roux-en-Y gastric bypass and have found it to be comparable to or superior to the Roux-en-Y gastric bypass. Our results have also indicated an excess body weight loss rate of $68.7 \%$ and $83.4 \%$ in 6 months and 1 year, respectively (See Table 2 for details), which is comparable to the data shown in the literature by high volume centers..$^{[2-7,11-16]}$ However, our results seem better since it is a single center study and a single surgeon performed the surgeries in less than two years.

\section{Complication Rates}

The procedure has relatively lower complication rates when compared to the Roux-en-Y gastric bypass. Lee et al. have compared laparoscopic single anastomosis gastric bypass to Roux-en-Y gastric bypass and shown that early postoperative rate is 3 times higher when compared to LSAGB(6). Usually, anastomosis rates following LSAGB have ranged between 0.06 to $5.6 \%$. Furthermore,mortality rates are below $1 \%$ in most studies. ${ }^{[2-7,11-16]}$ In our series,

there were no major complications, but there was minor deep vein thrombosis in a 60-year-old female patient and was treated conservatively.

\section{Duration of Hospitalization}

Mean duration of hospitalization is 1.5 days to 7 days in large patient volume reports. ${ }^{[2-7,11-16]}$ We believe that it depends on the anesthesia type, blood loss, and overall comorbidities of the patient. In our institution, we prefer keeping the patient until the postoperative third day following gastrografin swallow. We remove the nasogastric tube for leak test and discharge the patient usually on postoperative fourth day.

LSAGB is a safe procedure with shorter learning curve, and it is comparable to Roux-en-Y gastric bypass. Contrary to Roux-en-Y gastric bypass, LSAGB has a better safety profile. Therefore, LSAGB is an ideal bariatric and metabolic surgical procedure adapted by increasing number of surgeons.

\section{References}

1. Buchwald H, Buchwald JN. Evolution of operative procedures 
for the management of morbid obesity 1950-2000. Obes Surg 2002;12:705-17.

2. Rutledge $R$. The mini-gastric bypass: experience with the first 1,274 cases. Obes Surg 2001;11:276-80.

3. Rutledge $R$, Walsh TR. Continued excellent results with the mini-gastric bypass: six-year study in 2,410 patients. Obes Surg 2005;15:1304-8.

4. Wang W, Wei PL, Lee YC, Huang MT, Chiu CC, Lee WJ. Shortterm results of laparoscopic mini-gastric bypass. Obes Surg 2005; 15:648-54.

6. Carbajo M, García-Caballero M, Toledano M, Osorio D, García-Lanza C, Carmona JA. One-anastomosis gastric bypass by laparoscopy: results of the first 209 patients. Obes Surg 2005; 15:398-404.

6. Lee WJ, Yu PJ, Wang W, Chen TC, Wei PL, Huang MT. Laparoscopic Roux-en-Y versus mini-gastric bypass for the treatment of morbid obesity: a prospective randomized controlled clinical trial. Ann Surg 2005;242:20-8.

7. Noun R, Skaff J, Riachi E, Daher R, Antoun NA, Nasr M. One thousand consecutive mini-gastric bypass: short- and longterm outcome. Obes Surg 2012;22:697-703.

8. Kim MJ, Hur KY. Short-term outcomes of laparoscopic single anastomosis gastric bypass (LSAGB) for the treatment of type 2 diabetes in lower BMI $(<30 \mathrm{~kg} / \mathrm{m}(2))$ patients. Obes Surg 2014;24:1044-51.

9. Rutledge R, Kular KS, Manchanda N, Bhandari M, Goel R. A comparison of the outcomes revision of the roux-en- $Y$ (RNY) and mini-gastric bypass (MGB); hard vs. easy. Eur J Endosc Laparosc Surg 2014;1:1-6.

10. Musella M, Milone M, Gaudioso D, Bianco P, Palumbo R, Galloro $G$, et al. A decade of bariatric surgery. What have we learned? Outcome in 520 patients from a single institution. Int J Surg 2014;12 Suppl 1:S183-8.

11. Kular KS, Manchanda N, Rutledge R. Analysis of the fiveyear outcomes of sleeve gastrectomy and mini gastric bypass: a report from the Indian sub-continent. Obes Surg 2014;24:1724-8.

12. Kular KS, Manchanda N, Rutledge R. A 6-year experience with 1,054 mini-gastric bypasses-first study from Indian subcontinent. Obes Surg 2014;24:1430-5.

13. Musella M, Susa A, Greco F, De Luca M, Manno E, Di Stefano $C$, et al. The laparoscopic mini-gastric bypass: the Italian experience: outcomes from 974 consecutive cases in a multicenter review. Surg Endosc 2014;28:156-63.

14. Piazza L, Ferrara F, Leanza S, Coco D, Sarvà S, Bellia A, et al. Laparoscopic mini-gastric bypass: short-term single-institute experience. Updates Surg 2011;63:239-42.

15. Coskun H, Hasbahceci M, Bozkurt S, Yardımcı E, Cipe G, Malya FU, et al. Effect of laparoscopic mini-gastric bypass on diabetes in morbidly obese patients. Eur J Endosc Laparosc Surg 2014;1:40-4.

16. Ozmen MM, Sahin TT, Gundogdu E, Moran M, Isgenderova S. Mini gastric bypass on type 2 diabetes mellitus: fast, safe and effective. Obesity Surgery 2014;24:1257. 